\title{
A Threshold Voltage Model for the IMOS Device Using Hetero Structure
}

\author{
Heming $\mathrm{Yao}^{\mathrm{a}}$ \\ a Beihang Sino-French Engineer School, \\ Beihang University, \\ Beijing, China \\ yaoheming2010@yahoo.cn
}

\begin{abstract}
IMOS allows very sharp subthreshold slopes, even down to a few $\mathrm{mV} / \mathrm{dec}$ at room temperature. However, the device has serious reliability problems, such as large threshold voltage shifts, caused by hot carrier induced damage and the large supply voltage. Hetero IMOS, using the small bandgap material as the channel material, improves device reliability and decreases power consumption by reducing off-state currents and features a lower breakdown voltage compared to conventional IMOS. In this paper, a threshold voltage model is proposed in different distribution of surface electric field and in the condition of avalanche breakdown. The result is analyzed with the simulation data. It promotes the application of IMOS to VLSI design.
\end{abstract}

Keywords-avalanche breakdown; Hetero IMOS; threshold voltage

\section{INTRODUCTION}

While the feature size of MOSFET devices is reaching its physical limits due to several phenomena, such as the high subthreshold swing [1], the thermal diffusion of carriers at low gate voltages and the short channel effect [2], IMOS is receiving an increasing amount of attention. IMOS, proposeded for the first by Gopalakrishnan et al. [3] in 2002, is able to reach subthreshold slopes as small as $5 \mathrm{mV} / \mathrm{dec}$ at room temperature [4]. However, some problems of IMOS, such as the relatively high operating voltage and power consumption, are never ignored due to their negative influence on its reliability. A germanium based device was analyzed by Gopalakrishnan et al. to improve the performance of IMOS [5]. However, there are still many technological barriers to fabricate practical germanium devices [6]. Although a single gate IMOS structure [7], using SiGe-On-Insulator technology (SG-SGOI IMOS) and employing $\mathrm{Si}_{1-\mathrm{x}} \mathrm{Ge}_{\mathrm{x}}$ as a channel material, leads to significant reduction in supply voltages. However, this technique results into an increase in on-state current. Hetero IMOS [7], equipped with with $\mathrm{Si} / \mathrm{Si}-\mathrm{Ge}$ channel, has a lower breakdown voltage, and reduces the operating voltage and power consumption of devices.

In this paper, a model for threshold voltage of Hetero PIMOS is developed by resolving the $2 \mathrm{D}$ Poisson equation and in the condition of avalanche breakdown. By analyzing the model, the reliance of threshold voltage on the drainsource voltage, on the silicon-cap layer thicknesses and on the gate length is studied, respectively. The results of the models are in good agreement with that of simulation.

\section{HETERO IMOS STRUCTURE}

The basic device structure of the Hetero IMOS is a gated p-i-n diode Fig. 1, as that in the IMOS, except that the channel region employs the $\mathrm{Si} / \mathrm{Si}-\mathrm{Ge}$ channel. In this structure, on top of the $\mathrm{Si}_{1-\mathrm{x}} \mathrm{Ge}_{\mathrm{x}}$ layer is placed a silicon-cap layer. The overall thickness of this layer and the $\mathrm{Si}_{1-\mathrm{x}} \mathrm{Ge}_{\mathrm{x}}$ layer is equal to the thin-film thickness of the device.

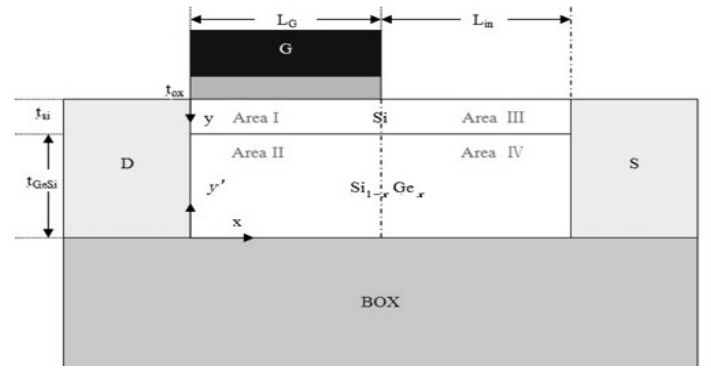

Figure1. Cross section of the Hetero P-IMOS

\section{Threshold Voltage Model}

The input parameters used for the Hetero P-IMOS are indicated in the Fig. 1. The gate voltage, which leads to the avalanche breakdown, is called the threshold voltage [8]. The distribution of the electric field is firstly analyzed to derive the threshold voltage model. The device is divided into four different parts Area I, Area II, Area III and Area IV. The threshold voltage of the IMOS is controlled by gate voltage and source-drain voltage, so the 2D Poisson should be used to describe the characteristics of Area I and Area II, before the strong inversion appears. The equations in the Area I and Area II can be written as:

$$
\begin{gathered}
\frac{\partial^{2} \phi_{1}(x, y)}{\partial x^{2}}+\frac{\partial^{2} \phi_{1}(x, y)}{\partial y^{2}}=-\frac{q N_{A}}{\mathcal{E}_{S i}}, \\
\frac{\partial^{2} \phi_{2}\left(x, y^{\prime}\right)}{\partial x^{2}}+\frac{\partial^{2} \phi_{2}\left(x, y^{\prime}\right)}{\left.\partial y^{\prime 2}, 0 \leqslant y \leqslant \mathrm{t}_{\mathrm{si}}\right)}=-\frac{q N_{A}}{\mathcal{E}_{G e S i}}, \\
\left(0 \leqslant \mathrm{x} \leqslant \mathrm{L}_{\mathrm{G}}, 0 \leqslant y^{\prime} \leqslant \mathrm{t}_{\mathrm{GeSi}}\right)
\end{gathered}
$$

The potential in the vertical direction in the channel region can be approximated by a parabolic function, the equations are as follows:

$$
\phi(x, y)=\phi_{S 1}(x)+c_{11}(x) y+c_{12}(x) y^{2}
$$




$$
\begin{gathered}
\left(0 \leqslant \mathrm{x} \leqslant \mathrm{L}_{\mathrm{c}}, 0 \leqslant y \leqslant \mathrm{t}_{\mathrm{si}}\right) \\
\phi_{2}\left(x, y^{\prime}\right)=\phi_{22}(x)+c_{21}(x) y^{\prime}+c_{22}(x) y^{2}, \\
\left(0 \leqslant \mathrm{x} \leqslant \mathrm{L}_{\mathrm{c}}, 0 \leqslant y^{\prime} \leqslant \mathrm{t}_{\mathrm{cesi}}\right)
\end{gathered}
$$

We hypothesize that 1) $t_{\mathrm{BOX}}>>t_{\mathrm{S}}$ and $\left.t_{\mathrm{BOX}}>>t_{\mathrm{GeSi}} ; 2\right)$ the Substrate electrode links to the ground; 3 ) The oxide layers contain no impurity charge. The equations $(1,2)$ can be solved, by using the following boundary conditions:

$$
\begin{aligned}
& {\left[\frac{d \phi_{1}(x, y)}{d y}\right]_{y=0}=-\frac{\varepsilon_{O X}}{\varepsilon_{S i}}\left(\frac{V_{G}^{\prime}-\phi_{S 1}(x)}{t_{O X}}\right)} \\
& {\left[\frac{d \phi_{2}\left(x, y^{\prime}\right)}{d y^{\prime}}\right]_{y^{\prime}=0}=0} \\
& {\left[\frac{d \phi_{1}(x, y)}{d y}\right]_{y=t_{S i}}=\left[-\frac{\varepsilon_{G e S i}}{\varepsilon_{S i}} \frac{d \phi_{2}\left(x, y^{\prime}\right)}{d y^{\prime}}\right]_{y^{\prime}=t_{C O S i}}} \\
& {\left[\phi_{1}(x, y)\right]_{y=t_{S i}}=\left[\frac{\varepsilon_{G e S i}}{\mathcal{E}_{S i}} \phi_{2}\left(x, y^{\prime}\right)\right]_{y^{\prime}=t_{G e S i}}}
\end{aligned}
$$

Substituting $\phi_{1}, \phi_{2}$ and substituting y $=0$, we obtain the equations:

$$
\begin{aligned}
& \frac{\partial^{2} \phi_{s 1}(x)}{\partial x^{2}}-a_{1} \phi_{s 1}(x)+a_{2} \phi_{s 2}(x)=b_{1} \\
& \frac{\partial^{2} \phi_{s 2}(x)}{\partial x^{2}}-a_{3} \phi_{s 1}(x)+a_{4} \phi_{s 2}(x)=b_{2}
\end{aligned}
$$

We substitute $\phi_{s 2}$ with $\phi_{s 1}$ in the equation (9), the result is as follows:

$$
\frac{\partial^{4} \phi_{s 1}(x)}{\partial x^{4}}-\left(a_{4}-a_{1}\right) \frac{\partial^{2} \phi_{s 1}(x)}{\partial x^{2}}-\left(a_{1} a_{4}+a_{2} a_{3}\right) \phi_{s 1}(x)=\left(a_{2} b_{2}-a_{4} b\right)
$$

In fact, the variation of $\phi_{s 1}(x)$ is enough so smooth that the equation (11) could be simplify as the equation (12):

$$
\frac{\partial^{2} \phi_{s 1}(x)}{\partial x^{2}}-\alpha \phi_{s 1}(x)=\beta
$$

We can acquire the solutions:

$$
\left.\phi_{s 1}(x)=A \exp \sqrt{\alpha} \alpha\right)+B \exp (\sqrt{\alpha} x)-\frac{\beta}{\alpha}
$$

The electric field distribution can be written as follows:

$$
E_{1}(x)=-A \sqrt{\alpha} \exp (\sqrt{\alpha} x)+B \sqrt{\alpha} \exp (-\sqrt{\alpha} x)
$$

As for Area III and Area IV, 1D Poisson equation will be now applied to determine $\phi_{s 3}, \phi_{s 4}$, we obtain the equations:

$$
\begin{aligned}
& \phi_{S 3}(x)=-\frac{q N_{A}}{2 \varepsilon_{S i}} x^{2}+C_{3} x+D_{3} \\
& \phi_{S 4}(x)=-\frac{q N_{A}}{2 \varepsilon_{G e S i}} x^{2}+C_{4} x+D_{4}
\end{aligned}
$$

The corresponding electric field distribution of the Area III can be written as follows:

$$
E_{3}(x)=\frac{q N_{A}}{\varepsilon_{S i}} x-C_{3}
$$

In order to determine the unknown element $\mathrm{A}, \mathrm{B}, \mathrm{C}$ and $\mathrm{D}$, the following boundary conditions are used:

$$
\left\{\begin{array}{l}
\phi_{S 1}(0)=V_{D} \\
\phi_{S 3}\left(L_{G}+L_{i n}\right)=V_{S}+V_{b i} \\
\phi_{S 1}\left(L_{G}\right)=\phi_{S 3}\left(L_{G}\right) \\
{\left[\frac{d \phi_{S 1}(x)}{d x}\right]_{x=L_{G}}=\left[\frac{d \phi_{s 3}(x)}{d x}\right]_{x=L_{G}}}
\end{array}\right.
$$

It has been proved that the avalanche breakdown happens firstly in the silicon-cap layer[3]. Therefore, to obtain a model for threshold voltage, we have to find the precise location of the maximum electric field from equations (14) and (17) by substituting the condition $\mathrm{dE} / \mathrm{dx}=0$. The maximum electric field location will be obtained as $\mathrm{x}_{\max }=\mathrm{L}_{\mathrm{G}}$. When the electric filed in $\mathrm{x}_{\max }=\mathrm{L}_{\mathrm{G}}$, reaches critical electric field, the avalanche breakdown is triggered, the device is open. By substituting $\mathrm{x}_{\max }=\mathrm{L}_{\mathrm{G}}$ into (17), and use $\mathrm{E}_{3}=\mathrm{E}_{\text {cri }}$, the threshold model is written as:

$$
\begin{aligned}
& V_{t h}=\frac{\left(a_{1}-a_{4}\right) \frac{\chi_{1}}{\chi_{2}}+\frac{a_{4} q N_{A}}{\varepsilon_{S i}}-\frac{a_{2} q N_{A}}{\varepsilon_{G e S i}}}{a_{4} \xi_{2}-a_{2} \xi_{1}}-\Delta \varphi_{M I} \\
& E_{c r i}=\frac{q N_{A} L_{G}}{\varepsilon_{S i}}-C_{3}
\end{aligned}
$$

When we have:

$$
\begin{aligned}
& \chi_{1}=\left(r_{2}-r_{1}\right) V_{D} \exp \left(\sqrt{\alpha} L_{G}\right)+\frac{r_{2} q N_{A}}{\varepsilon_{S i}}\left(L_{i n}^{2}+2 L_{G} L_{i n}\right)- \\
& \frac{r_{1} q N_{A} L_{G}}{\sqrt{\alpha} \varepsilon_{S i}}+\frac{C_{3} r_{1}}{\sqrt{\alpha}}-r_{2} C_{3} L_{i n}+r_{2}\left(V_{S}+V_{b i}\right) \\
& \chi_{2}=\frac{r_{1} \exp \left(\sqrt{\alpha} L_{G}\right)}{\alpha}-\frac{r_{2}}{\alpha}-\frac{r_{2} \exp \left(\sqrt{\alpha} L_{G}\right)}{\alpha} \\
& \xi_{1}=\frac{2 \frac{\varepsilon_{O X} t_{S i}}{\varepsilon_{S i} t_{o X}}+\frac{\varepsilon_{o X} t_{G e S i}}{\varepsilon_{G e S i} t_{o X}}}{t_{S i}^{2}\left(1+\frac{\varepsilon_{S i} t_{G e S i}}{\varepsilon_{G e S i} t_{S i}}\right)}, \xi_{2}=\frac{\frac{\varepsilon_{O X} t_{S i}}{\varepsilon_{S i} t_{o X}}}{t_{G e S i}^{2}\left(1+\frac{\varepsilon_{G e S i} t_{S i}}{\varepsilon_{S i} t_{G e S i}}\right)},
\end{aligned}
$$




$$
\begin{aligned}
& \alpha=\frac{a_{2} a_{3}-a_{1} a_{4}}{a_{1}-a_{4}}, \beta=\frac{c_{2} a_{2}-c_{1} a_{4}}{a_{1}-a_{4}} \\
& a_{1}=\frac{2+\frac{2 \varepsilon_{O X} t_{S i}}{\varepsilon_{S i} t_{O X}}+\frac{\varepsilon_{O X} t_{G e S i}}{\varepsilon_{G e S} t_{o x}}}{t_{S i}^{2}\left(1+\frac{\varepsilon_{S i} t_{G e S i}}{\varepsilon_{G e S} t_{S i}}\right)}, a_{2}=\frac{2}{t_{S i}^{2}\left(1+\frac{\varepsilon_{S i} t_{G e S i}}{\boldsymbol{\varepsilon}_{G e S} t_{S i}}\right)}, \\
& a_{3}=\frac{2+\frac{\varepsilon_{o x} t_{S i}}{\varepsilon_{S i} t_{o x}}}{t_{G e S i}^{2}\left(1+\frac{\varepsilon_{G e S} t_{S i}}{\varepsilon_{S i} t_{G e S i}}\right)}, a_{4}=\frac{2}{t_{G e S i}^{2}\left(1+\frac{\varepsilon_{G e S} t_{S i}}{\varepsilon_{S i} t_{G e S i}}\right)} \\
& c_{1}=\frac{q N_{A}}{\mathcal{E}_{S i}}-\frac{2 \frac{\mathcal{\varepsilon}_{O X} t_{S i}}{\boldsymbol{\varepsilon}_{S i} t_{o X}}+\frac{\boldsymbol{\varepsilon}_{o X} t_{G e S i}}{\varepsilon_{G e S i} t_{o X}}}{t_{S i}^{2}\left(1+\frac{\boldsymbol{\varepsilon}_{S i} t_{G e S i}}{\boldsymbol{\varepsilon}_{G e S i} t_{S i}}\right)} V_{G}^{\prime}, \\
& c_{2}=\frac{q N_{A}}{\varepsilon_{G e S i}}-\frac{\frac{\boldsymbol{\varepsilon}_{O X} t_{S i}}{\boldsymbol{\varepsilon}_{S i} t_{O X}}}{t_{G e S i}^{2}\left(1+\frac{\boldsymbol{\varepsilon}_{G e S i} t_{S i}}{\boldsymbol{\varepsilon}_{S i} t_{G e S i}}\right)} V_{G}^{\prime} \\
& V_{G}^{\prime}=V_{G}+\Delta \varphi_{M I}
\end{aligned}
$$

\section{Simulation Of Voltage Model}

To verify the accuracy of the threshold voltage model, we use Matlab for numerical analysis and use ISE to simulate the model proposed in the previous section.

The parameters used are as following: Work function of gate material $\Delta \varphi_{\mathrm{MI}}=4.6 \mathrm{eV}, \mathrm{T}=300 \mathrm{~K}$, Drain bias $\mathrm{V}_{\mathrm{D}}=0$, the source/drain/intrinsic doping $\mathrm{N}_{\mathrm{s}}=\mathrm{N}_{\mathrm{d}}=10^{20} \mathrm{~cm}^{-3}, \mathrm{~N}_{\mathrm{A}}=2 \times$ $10^{20} \mathrm{~cm}^{-3}$. The results are presented as the following figures.

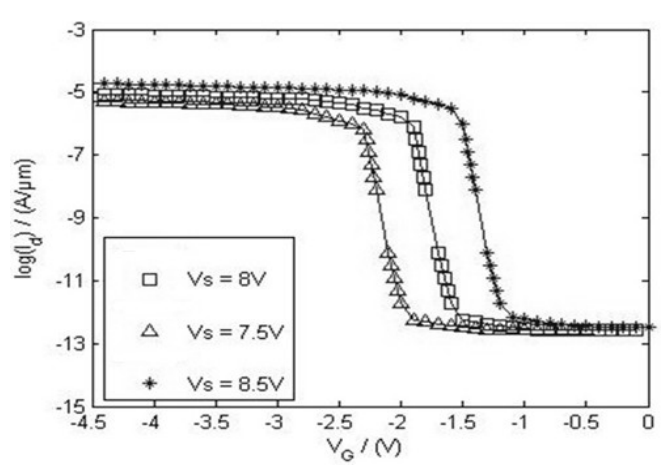

Figure 2. Transfer characteristic curve of Hetero P-IMOS

Fig. 2 presents the transfer characteristic curve of Hetero P-IMOS under different source voltage, including $\mathrm{L}_{\mathrm{G}}=\mathrm{L}_{\mathrm{in}}=$ $100 \mathrm{~nm}$. We can easily spot the influence of the source voltage on the threshold voltage. When $\mathrm{V}_{\mathrm{S}}$ reduces, the device needs the higher $\left|\mathrm{V}_{\mathrm{G}}\right|$ so as to attain the critical point of the avalanche breakdown.

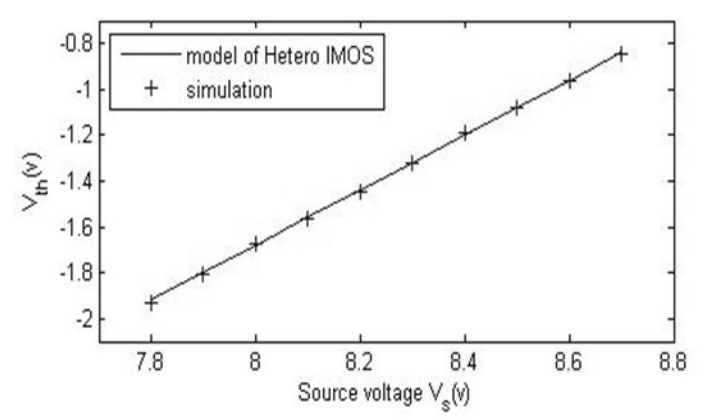

Figure 3. Variation of threshold voltage with Source voltage

Fig.3 shows the variation of threshold voltage with Source voltage. It can be found that simulation results and calculated results of model agree very well with each other. We can observe that $\left|V_{\text {th }}\right|$ increases when $V_{S}$ decreases.

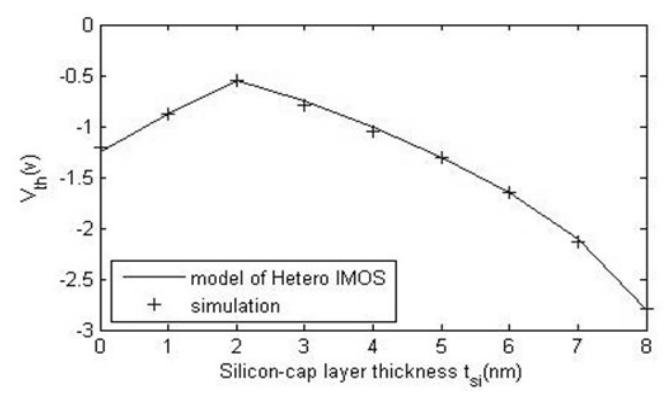

Figure 4. Variation of threshold voltage with $t_{\mathrm{si}}$

Threshold voltage $\left(\mathrm{V}_{\mathrm{th}}\right)$ as a function of the silicon-cap layer thicknesses is shown in Fig.4. By increasing $t_{s i}$, the electric field in the buried channel is decreased. Thus the ionization rate decreases and avalanche breakdown occurs at higher gate voltages. As a result, $\mathrm{V}_{\text {th }}$ increases. An optimum 
silicon-cap layer thickness equal to $2 \mathrm{~nm}$ is obtained for the structure.

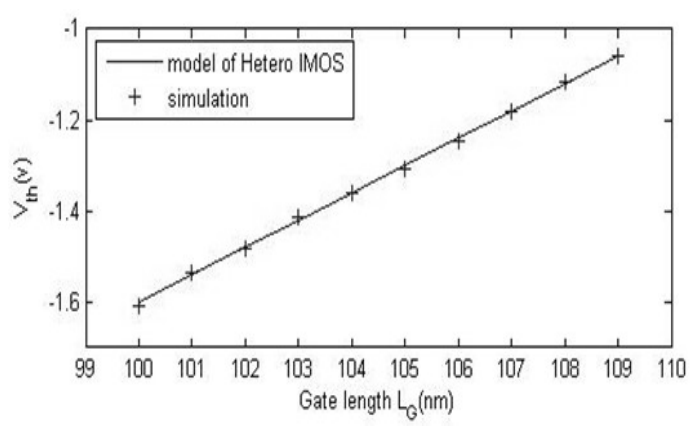

Figure 5. Variation of threshold voltage with the gate length

From Fig. 5 we can see that for a given polarization $\mathrm{Vs}=$ $7.8 \mathrm{~V}$, the threshold voltage $\left|\mathrm{V}_{\mathrm{th}}\right|$ is smaller for higher $\mathrm{L}_{\mathrm{G}} / \mathrm{L}_{\text {in }}$ ratios. However, the higher $\mathrm{L}_{\mathrm{G}} / \mathrm{L}_{\text {in }}$ is, the higher subthreshold slope is. Besides, with a higher $\mathrm{L}_{\mathrm{G}} / \mathrm{L}_{\text {in }}$, a bigger part of channel is controlled by the gate. Thus, the ionization rate and avalanche breakdown occurs at a smaller $\left|\mathrm{V}_{\mathrm{th}}\right|$.

\section{CONCLUSION}

A threshold voltage model of Hetero IMOS is proposed in this paper. The results of the model are in good agreement with that of simulation. The model in the paper can be easily used for the analysis and design of IMOS so that it is utilized to VILS design..

\section{REFERENCES}

[1] Choi W Y, Choi B Y, Woo D S, Lee J D, Park B G, "Reverse-order source/drain formation with double offset spacer (RODOS) for lowpower and high-speed application”, 2003 IEEE Trans. Nanotechnol 2 210

[2] Zheng X H, Huang A P, Yang Z C, Xiao Z S, Wang M, Cheng G A, "Diffusion behavior of dual capping layers in TiN/LaN/AlN/HfSiOx/Si stack", 2011 Acta Phys. Sin. 60017702 (in Chinese)

[3] K. Gopalakrishnan, P. B. Gri_n, and J. D. Plummer, "I-MOS: A novel semiconductor device with a subthreshold slope lower than $\mathrm{kT} / \mathrm{q}$ ", IEDM, 2002. pp. 289-292.

[4] F. Mayer, C. Le Royer, G. Le Carval, L. Clavelier, and S. Deleonibus, "Static and Dynamic TCAD Analysis of IMOS Performance: From the Single Device to the Circuit," IEEE Trans. Electron Devices, vol. 53, no. 8, pp. 1852-1857, Aug. 2006.

[5] K. Gopalakrishnan, "Impact-Ionization MOS and its Derivatives for Logic and Memory", Thesis, 2004.

[6] K. Gopalakrishnan, R. Woo, C. Jungemann, P. B. Griffin, and J. D. Plummer, "Impact Ionization MOS (I-MOS)-Part I: Device and Circuit simulations, Trans. Electron Devices”, pp. 69-76, Jan. 2005.

[7] Hamed Nematian, "Reducing Breakdown Voltages in Impact Ionization Metal-Oxide-Semiconductor (I-MOS) Devices Using Hetero Structure", IEEE. 2008. pp.114-117.

[8] Li Yu-Chen, Zhang He-Ming "A analytic model for the thresholdvoltage of novel high-speed semiconductor device IMOS", Acta Phys. Sin. 2012. Vol. 61, No. 4, pp1-5. 